\title{
A THEOREM ON ORTHOGONAL FUNCTIONS WITH AN APPLICATION TO INTEGRAL INEQUALITIES*
}

BY

\author{
LLOYD L. DINES
}

It is well known that for a given finite set of functions

$\left\{f_{i}\right\}$

$$
f_{1}(x), f_{2}(x), \cdots, f_{m}(x),
$$

continuous on an interval

$$
a \leqq x \leqq b,
$$

a continuous function $f(x)$ can be determined which is orthogonal to all functions of the set, that is, the conditions

$$
\int_{a}^{b} f(x) f_{i}(x) d x=0 \quad(j=1,2, \cdots, m)
$$

can be satisfied. The principal object of the present paper is to determine under what conditions such a function $f(x)$ can be everywhere positive. This object is attained in the following

TheOrem I. A necessary and sufficient condition that a set of functions, continuous and linearly independent on a closed interval, admit a positive continuous function orthogonal to all of them is that every linear combination of the functions change sign on the interval. $\dagger$

In the last section of the paper an application of this theorem is given in a study of the integral inequality

$$
\phi(x)+\int_{a}^{b} \kappa(x, s) \phi(s) d s>0 .
$$

The principal result in this connection is the following

* Presented to the Society, San Francisco Section, under different title, June 12, 1926; received by the editors in November, 1926.

† This theorem is an analogue of an algebraic theorem given in a recent paper, Note on certain associated systemis of linear equalities and inequalities, Annals of Mathematics, (2), vol. 28 (1926-27), pp. $41-42$. 
THEOREM II. A necessary and sufficient condition that the integral inequality (1) admit a solution $\phi(x)$ is that every non-trivial solution $\psi(x)$ of the associated integral equation

$$
\psi(x)+\int_{a}^{b} \psi(s) \kappa(s, x) d s=0
$$

shall change sign.*

The first part of the paper is devoted to preliminary notions which are used in the proof of Theorem I.

1. An outline of the proof of Theorem I. The necessity of the condition in Theorem I is almost obvious. For if $f(x)$ is orthogonal to each of the functions $f_{i}(x)$, it is orthogonal to every linear combination of them, that is

$$
\int_{a}^{b} f(x) \sum_{j=1}^{m} a_{i} f_{j}(x) d x=0 ;
$$

and this relation for a positive $f(x)$ demands that the second factor of the integrand change sign unless it is identically zero. Hence since the functions are linearly dependent, the condition of the theorem is necessary.

To prove the sufficiency of the condition, we proceed as follows. By a certain well defined operation, the given set of $m$ functions $\left\{f_{i}\right\}$ is replaced by another set of $m$ functions called a reduced set. $\dagger$ The efficacy of the reduction is due to the following two properties:

(a) One function of the reduced set is identically zero.

(b) If the reduced set admits a positive function orthogonal to all of its members, the same is true of the original set.

The reduction process may be repeated, the property (b) persisting, and the property (a) introducing a new zero function at each repetition; so that after $m-1$ reductions the resulting set contains only one function which is different from zero. It is not difficult to show that this function admits a positive orthogonal function, whence from (b) it follows that the given set admits a positive orthogonal function.

A complication arises from the fact that the range of the argument of a "reduced" set of functions is not the same as the range of the argument of the original set. The functions of the reduced set are as a matter of fact functions of a variable whose range is a composite range.

\footnotetext{
- For an analogous algebraic theorem, see papers by Carver, Annals of Mathematics, (2), vol. 23 , p. 212; and the author, ibid., vol. 27, p. 57.

+ A reduction process for a set of functions on a general range has been described by the author in an earlier paper, $O n$ sets of functions of a general variable, these Transactions, vol. 29 (1927), pp. 463-470.
} 
2. Reduction and composition of a range relative to a function on it. Let us consider any continuous function $\rho(x)$ on the range

$$
a \leqq x \leqq b,
$$

which changes sign on the range. Relative to the function $\rho(x)$, we may determine two subclasses of the range $\mathfrak{X}$, defined as follows:

$$
\begin{aligned}
& \mathfrak{X}_{P}(\rho) \equiv[\text { all } x \text { such that } \rho(x) \geqq 0], \\
& \mathfrak{X}_{N}(\rho) \equiv[\text { all } x \text { such that } \rho(x)<0] .
\end{aligned}
$$

For convenience we denote the elements of these subclasses by $p^{(p)}$ and $n^{(p)}$ respectively; thus

$$
\mathfrak{X}_{P}^{(\rho)} \equiv\left[p^{(\rho)}\right], \quad \mathfrak{X}_{N}^{(\rho)} \equiv\left[n^{(\rho)}\right] .
$$

From the properties of continuous functions we may draw the following conclusions with reference to these subclasses. The subclass $\mathfrak{X}_{N}{ }^{(\rho)}$ consists (geometrically speaking) of linear intervals open at both ends except when terminated by an end point $a$ or $b$ of the fundamental interval $\mathfrak{X}$. The subclass $\mathfrak{X}_{P}{ }^{(p)}$ is a closed point set. If the reducing function $\rho(x)$ is of simple type (for example if it changes sign at each point at which it vanishes), $\mathfrak{X}_{P}{ }^{(p)}$ consists of closed linear intervals; and it will in fact always contain at least one such interval.*

From the two subclasses (2) we now form a composite range

$$
\mathfrak{X}^{(\rho)} \equiv \mathfrak{X}_{P}^{(\rho)} \mathfrak{X}_{N}^{(\rho)},
$$

the elements of which are bipartite, of the form $p^{(\rho)} n^{(\rho)}$. The new range

$$
\mathfrak{X}^{(\rho)} \equiv\left[x^{(\rho)}\right] \equiv\left[p^{(\rho)} n^{(\rho)}\right]
$$

is a two-dimensional point set contained in the square $\mathfrak{X}$. If $\rho(x)$ is of a simple type, the points constitute a set of rectangles forming a sort of irregular checker-board arrangement. And for any $\rho(x)$, the range will include at least one such rectangle.

A function on the range $\mathfrak{X}^{(\rho)}$ will be said to be continuous if it is a continuous function of the two variables $p^{(\rho)}, n^{(\rho)}$. A function will be said to

* But since $\mathfrak{X}_{p}^{(\rho)}$ includes the zeros of $\rho(x)$, it may contain isolated points, and even perfect sets of points which comprise no interval, as in the following example. Let $\mathfrak{X}$ be the closed interval $(0,2)$. On the left half of this interval form the Cantor perfect set by the removal of open middle thirds (see Pierpont's Theory of Functions of Real Variables, vol. I, \& 272). On this perfect set let $\rho(x)=0$, and on its complement with respect to $(0,1)$ let $\rho(x)$ be negative. For $x>1$ let $\rho(x)$ be positive. This interesting example was suggested to me by Professor Kellogg, to whom I am indebted for a number of valuable criticisms and suggestions. 
change sign internally on the range $\mathfrak{X}^{(\rho)}$ if it is positive at some inner points and negative at other inner points of the range. Analogous definitions of continuity and internal change of sign are to be understood relative to all the composite ranges occurring in what follows.

Suppose now that on the new composite range $\mathfrak{X}^{(\rho)}$ there is defined in any way a real, single-valued, continuous function $\sigma$, which changes sign internally on the range. Then this function determines two subclasses of the range $\mathfrak{X}^{(\rho)}$ :

$$
\begin{aligned}
& \mathfrak{X}_{P}^{(\rho \sigma)} \equiv\left[\text { all } x^{(\rho)} \text { for which } \sigma \geqq 0\right], \\
& \mathfrak{X}_{N}^{(\rho \sigma)} \equiv\left[\text { all } x^{(\rho)} \text { for which } \sigma<0\right] ;
\end{aligned}
$$

and from these subclasses we may form a composite class

$$
\mathfrak{X}^{(\rho \sigma)} \equiv \mathfrak{X}_{P}^{(\rho \sigma)} \mathfrak{X}_{N}^{(\rho \sigma)} .
$$

The elements of this class are quadripartite. Geometrically they form a point set in the four-dimensional hypercube $\mathfrak{X X X}$, of which some subset at least are inner points.

The process of reduction and composition can be repeated indefinitely, provided at each stage a reducing function is available. To generalize the procedure and notation, suppose reduction with respect to the successive reducing functions $\rho_{1}, \rho_{2}, \cdots, \rho_{k-1}$ has yielded the composite range

$$
\mathfrak{X}^{\left(\rho_{1} \rho_{2} \cdots \rho_{k}-1\right)},
$$

consisting of $2^{k-1}$-partite elements. Suppose turther that $\rho_{k}$ is a singlevalued continuous function changing sign internally on this range. By reduction with respect to $\rho_{k}$ and composition we obtain the new range

$$
\mathfrak{X}^{\left(\rho_{1} \rho_{2} \cdots \rho_{k}\right)} \equiv \mathfrak{X}_{P}^{\left(\rho_{1} \rho_{2} \cdots \rho_{k}\right)} \mathfrak{X}_{N}^{\left(\rho_{1} \rho_{2} \cdots \rho_{k}\right)} \equiv\left[x^{\left(\rho_{1} \rho_{2} \cdots \rho_{k}\right)}\right],
$$

the elements of which are $2^{k}$-partite. Geometrically the new range is a point set in the $2^{k}$-dimensional cube $\mathfrak{X X} \ldots \mathfrak{X}$, of which some points at least are inner points.

3. Reduced outer multiplication. Consider again the original range $\mathfrak{X}$ and a continuous reducing function $\rho(x)$ changing sign on $\mathfrak{X}$. This function determines with any second continuous function $f(x)$ a function on the composite range $\mathfrak{X}^{(\rho)}$, which we shall call their reduced outer product and denote by $((\rho f))$. Its functional values are defined by the formula

$((\rho f)) \quad \rho(p) f(n)-f(p) \rho(n), \quad(p, n)$ on $\mathfrak{X}_{P}(\rho) \mathfrak{X}_{N}^{(\rho)}$. 
This multiplication is clearly not commutative. Its most obvious property is that $((\rho \rho))=0$. Another property easily verified is that $((\rho f))$ is continuous on $\mathfrak{X}_{P}{ }^{(\rho)} \mathfrak{X}_{N}(\rho)$.

Reduced outer multiplication is defined in a similar way upon any of the composite ranges described in the preceding section. Consider for example the reduced composite range

$$
\mathfrak{X}^{\left(\rho_{1} \rho_{2} \cdots \rho_{k-1}\right)} \text {, }
$$

and suppose that $\rho_{k}$ is a continuous function changing sign on this range. Then $\rho_{k}$ determines with any second continuous function $f_{k}$ on the range a reduced outer product $\left(\left(\rho_{k} f_{k}\right)\right)$, given by the formula

$\left(\left(\rho_{k} f_{k}\right)\right) \quad \rho_{k}(p) f_{k}(n)-f_{k}(p) \rho_{k}(n), \quad(p, n)$ on $\mathfrak{X}_{P}\left(\rho_{1} \rho_{2} \cdots \rho_{k}\right) \mathfrak{X}_{N}\left(\rho_{p_{1} \rho_{2}} \cdots \rho_{k}\right)$.

This product is continuous at all points of its region of definition.

4. Reduction of a set of functions. Consider the set of functions

$$
f_{1}(x), f_{2}(x), \cdots, f_{m}(x),
$$

continuous on the range $\mathfrak{X}$, and suppose that $f_{1}(x)$ changes sign.

Relative to $f_{1}(x)$ we form a new set of $m$ functions

$$
\left\{f_{i}^{(1)}\right\} \quad\left(\left(f_{1} f_{1}\right)\right),\left(\left(f_{1} f_{2}\right)\right), \cdots,\left(\left(f_{1} f_{m}\right)\right),
$$

each of which is the reduced outer product of the corresponding function of the given set by $f_{1}$. All functions of this reduced set are on the composite range $\mathfrak{X}^{\left(f_{1}\right)}$, and are continuous on that range. It is to be noted particularly that the first function $\left(\left(f_{1} f_{1}\right)\right)$ is identically zero. (See property (a) of $\$ 1$.)

The reduction process may now be repeated, the second function $f_{2}^{(1)}$ $\left(\equiv\left(\left(f_{1} f_{2}\right)\right)\right)$ being used as a reducing function (assuming that it changes sign), and a second reduced set of functions obtained, having the property that its first two functions are identically zero. Its range $\mathfrak{X}_{\mathcal{U}_{\mathcal{L}_{2}}}^{\left({ }^{(1)}\right)}$ will be denoted for brevity by $\mathfrak{X}^{(12)}$.

To make the reduction procedure and notation general, let $\left.\left\{f_{i}^{(12} \cdots k-1\right)\right\}$ denote the set of functions obtained by $k-1$ successive reductions of the kind indicated. Its first $k-1$ functions are identically zero, and its $k$ th function is $f_{k}^{(12 \cdots k-1)}$. If this function changes sign we may use it as a reducing function and form the new set $\left\{f_{i}^{(12 \cdots k)}\right\}$, each function of which is the reduced outer product of the corresponding function of $\left\{f_{i}^{(12 \cdots k-1)}\right\}$ by $f_{k}^{(12 \cdots k-1)}$. The functions of this new set are defined and continuous on the range $\mathfrak{X}^{(12 \cdots k)}$, and the first $k$ of them are identically zero. 
The reduction procedure thus defined will after $m-1$ operations yield a set of functions all of which except the last are identically zero. The assumption which we have made that at each stage the reducing function changes sign will now be justified.

LEMMA I. If every linear combination

$$
a_{1} f_{1}(x)+a_{2} f_{2}(x)+\cdots+a_{m} f_{m}(x)
$$

of the set of functions $\left\{f_{i}(x)\right\}$ changes sign on $\mathfrak{X}$, then every linear combination

$$
a_{2} f_{2}^{(1)}+a_{3} f_{3}^{(1)}+\cdots+a_{m} f_{m}^{(1)}
$$

of the last $m-1$ functions of the reduced set $\left\{f_{i}^{(1)}\right\}$ changes sign internally on its range $\mathfrak{X}^{(1)}$.

To prove the proposition indirectly, suppose that there is a set of constant multipliers $a_{2}, a_{3}, \cdots, a_{m}$, such that

$$
\sum_{j=2}^{m} a_{i} f_{i}^{(1)} \geqq 0 \quad \text { on } \mathfrak{X}^{(1)} .
$$

We first recall that the range $\mathfrak{X}^{(1)}$ is a composite range, $\mathfrak{X}^{(1)} \equiv \mathfrak{X}_{P^{(1)}} \mathfrak{X}_{N^{(1)}}$, the first component class $\mathfrak{X}_{P}{ }^{(1)}$ consisting of those elements of $\mathfrak{X}$ for which $f_{1}$ is positive or zero. For our present purpose, it is convenient to divide the class $\mathfrak{X}_{P}^{(1)}$ into two subclasses:

$$
\mathfrak{X}_{P}^{(1)} \equiv \mathfrak{X}_{P}{ }^{(1)}+\mathfrak{X}_{Z^{(1)}},
$$

where

$$
\begin{aligned}
\mathfrak{X}_{P}^{\prime(1)} & \equiv\left[\text { all } x \text { for which } f_{1}>0\right] \\
\mathfrak{X}_{Z}^{(1)} & \equiv\left[\text { all } x \text { for which } f_{1}=0\right] .
\end{aligned}
$$

Next, recalling the definition of the reduced function $f_{i}^{(1)}$, we may replace our supposition (3) by the two statements

$$
\begin{array}{rlr}
\sum_{j=2}^{m} a_{j}\left[f_{1}\left(p^{\prime}\right) f_{j}(n)-f_{j}\left(p^{\prime}\right) f_{1}(n)\right] & \geqq 0 & \left(p^{\prime}, n\right) \text { on } \mathfrak{X}_{P}^{\prime(1)} \mathfrak{X}_{N}^{(1)}, \\
-\sum_{j=2}^{m} a_{j} f_{i}(\mathbf{z}) f_{1}(n) & \geqq 0 & (z, n) \text { on } \mathfrak{X}_{Z}^{(1)} \mathfrak{X}_{N}^{(1)} .
\end{array}
$$

Now since $-f_{1}\left(p^{\prime}\right) f_{1}(n)$ is certainly positive, we may obtain from (4) the equivalent statement 


$$
\sum_{j=2}^{m} a_{j} \frac{f_{j}\left(p^{\prime}\right)}{f_{1}\left(p^{\prime}\right)} \geqq \sum_{j=2}^{m} a_{j} \frac{f_{j}(n)}{f_{1}(n)} \quad p^{\prime} \text { on } \mathfrak{X}_{P}{ }^{(1)}, n \text { on } \mathfrak{X}_{N}{ }^{(1)} \text {. }
$$

The various values of the expression on the left side of (6) must have a greatest lower bound, and those of the expression on the right must have a least upper bound, which bounds may or may not coincide.

In any case we may choose a constant $a_{1}$ such that

$$
\sum_{j=2}^{m} a_{j} \frac{f_{j}\left(p^{\prime}\right)}{f_{1}\left(p^{\prime}\right)} \geqq-a_{1} \geqq \sum_{j=2}^{m} a_{j} \frac{f_{j}(n)}{f_{1}(n)} \quad p^{\prime} \text { on } \mathfrak{X}_{P}{ }^{\prime(1)}, n \text { on } \mathfrak{X}_{N}{ }^{(1)} .
$$

And from this double relation we obtain

$$
\sum_{j=1}^{n} a_{j} f_{j}\left(p^{\prime}\right) \geqq 0, \quad \sum_{j=1}^{n} a_{j} f_{j}(n) \geqq 0 \quad \quad p^{\prime} \text { on } \mathfrak{X}_{P}{ }^{(1)}, n \text { on } \mathfrak{X}_{N}^{(1)} \text {. }
$$

Furthermore, division of (5) by $-f_{1}(n)$, which is certainly positive, yields a statement which may be written

$$
\sum_{j=1}^{m} a_{j} f_{j}(z) \geqq 0 \quad \text { zon } \mathfrak{X}_{z}^{(1)}
$$

But the statements (7) and (8) can be combined to give

$$
\sum_{j=1}^{m} a_{j} f_{i}(x) \geqq 0
$$

$x$ on $\mathfrak{X}$.

This contradicts the hypothesis of the lemma, and the contradiction proves that every linear combination $\sum_{j=2}^{m} a_{i} f_{j}^{(1)}$ must be negative somewhere on $\mathfrak{X}^{(1)}$. To see that it must be negative at an inner point, we note first that all points of $\mathfrak{X}_{P}^{\prime}{ }_{P}^{(1)} \mathfrak{X}_{N}{ }^{(1)}$ are inner points. If it is negative at none of these points, then it must be negative on $\mathfrak{X}_{Z^{(1)}} \mathfrak{X}_{N}^{(1)}$, that is, the left side of (5) must be somewhere negative, and hence the left side of (8) must be negative at some point of $\mathfrak{X}_{z^{(1)}}$. If the left side of (8) is negative at an inner point of $\mathfrak{X}_{z}{ }^{(1)}$, then the left side of (5) is negative at an inner point of $\mathfrak{X}_{Z^{(1)}} \mathfrak{X}_{N}{ }^{(1)}$ which is a fortiori an inner point of $\mathfrak{X}^{(1)}$, and our desired conclusion is reached. If on the other hand the left side of (8) is negative only at frontier points of $\mathfrak{X}_{z}{ }^{(1)}$, it follows from continuity (since each such frontier point is a limit point of $\mathfrak{X}_{P}^{\prime}{ }^{(1)}$ or $\mathfrak{X}_{N}^{(1)}$ ) that one of the inequalities in (7) is contradicted. But this involves a contradiction of (4), which contradiction means explicitly that $\sum_{j=2}^{m} a_{j} f_{j}^{(1)}$ is negative on $\mathfrak{X}_{P}^{\prime(1)} \mathfrak{X}_{N}^{(1)}$, hence at an inner point of $\mathfrak{X}^{(1)}$. In an entirely analogous way it may be shown that every such linear combination must be positive at an inner point of $\mathfrak{X}^{(1)}$. The proof of the lemma is then complete. 
An argument similar to the one we have just made suffices to prove the following

LEMMA II. If every linear combination

$$
a_{k} f_{k}^{(12 \cdots k-1)}+a_{k+1} f_{k+1}^{(12 \cdots k-1)}+\cdots+a_{m} f_{m}^{(12 \cdots k-1)}
$$

of the last $m-k+1$ functions of the reduced set $\left\{f_{i}^{(12 \cdots k-1)}\right\}$ changes sign on its range $\mathfrak{X}^{(12 \cdots k-1)}$, then every linear combination

$$
a_{k+1} f_{k+1}^{(12 \cdots k)}+a_{k+2} f_{k+2}^{(12 \cdots k)}+\cdots+a_{m} f_{m}^{(12 \cdots k)}
$$

of the last $m-k$ functions of the reduced set $\left\{f_{i}^{(12 \cdots k)}\right\}$ changes sign internally on its range $\mathfrak{X}^{(12 \cdots k)}$.

Successive application of these lemmas now justifies the assumption which we made in the early part of this section:

If every linear combination of the given set of functions changes sign on $\mathfrak{X}$, then every function appearing as a reducing function in the progressive reduction process described in this section changes sign internally on its range.

5. Proof of property (b) of $\S 1$. Two functions $f$ and $g$ defined upon one of the reduced composite ranges $\mathfrak{X}^{(12 \cdots k)}$, will be said to be orthogonal one to the other if*

$$
\int_{\mathfrak{X}^{(12 \ldots k)}} f g=0 \text {. }
$$

Suppose now that $k-1$ reductions of the given set of functions

have yielded the set

$$
f_{1}, f_{2}, \cdots, f_{m}
$$

$$
0, \cdots, 0, f_{k}^{(12 \cdots k-1)}, f_{k+1}^{(12 \cdots k-1)}, \cdots, f_{m}^{(12 \cdots k-1)},
$$

on the range $\mathfrak{X}^{(12 \cdots k-1)}$, and the reduction of this latter set with respect to $f_{k}^{(12 \cdots k-1)}$ has yielded the set

$$
0, \cdots, 0, f_{k+1}^{(12 \cdots k)}, f_{k+2}^{(12 \cdots k)}, \cdots, f_{m}^{(12 \cdots k)},
$$

on the range $\mathfrak{X}^{(12 \cdots k)}$.

- In this section and in those which follow, the integrals may be taken in the sense of Lebesgue whenever there is doubt as to their existence in the sense of Riemann. Of their existence in the former sense there will be no doubt. 
Suppose further that relative to this last set $\left\{f_{i}^{(12 \cdots k)}\right\}$ there is a function $\Pi^{(12 \cdots k)}$, everywhere positive and continuous on the range $\mathfrak{X}^{(12 \cdots k)}$, and orthogonal to all functions of the set.

Then there is a function $\Pi^{(12 \cdots k-1)}$, everywhere positive on the preceding range $\mathfrak{X}^{(12 \cdots k-1)}$, which is orthogonal to all functions of the preceding set $\left\{f_{i}^{(12 \cdots k-1)}\right\}$.

To prove this proposition we start with the hypothesis that the positive function $\Pi^{(12 \cdots k)}$ satisfies the conditions

$$
\int_{\mathfrak{X}^{(12 \cdots k)}} \Pi^{(12 \cdots k)} f_{j}^{(12 \cdots k)}=0 \quad(j=1,2, \cdots, m) .
$$

Since the defining formula for $f_{i}^{(12 \cdots k)}$ is

$$
\begin{array}{r}
f_{k}^{(12 \cdots k-1)}(p) f_{j}^{(12 \cdots k-1)}(n)-f_{i}^{(12 \cdots k-1)}(p) f_{k}^{(12 \cdots k-1)}(n) \\
(p, n) \text { on } \mathfrak{X}_{P}^{(12 \cdots k)} \mathfrak{X}_{N}^{(12 \cdots k),}
\end{array}
$$

we obtain, by substitution in (9) and decomposition of multiple integrals, the equalities

$$
\begin{array}{r}
\int_{\mathfrak{X}_{N}^{(12 \cdots k)}}\left[\int_{\mathfrak{X}_{P}^{(12 \cdots k)}} \Pi^{(12 \cdots k)}(p, n) f_{k}(12 \cdots k-1)(p) d p\right] f_{j}^{(12 \cdots k-1)}(n) d n \\
-\int_{\mathfrak{X}_{P}^{(12 \cdots k)}}\left[\int_{\mathfrak{X}_{N}^{(12 \cdots k)}} \Pi^{(12 \cdots k)}(p, n) f_{k}^{(12 \cdots k-1)}(n) d n\right] \\
\cdot f_{j}^{(12 \cdots k-1)}(p) d p=0 \quad(j=1,2, \cdots, m) .
\end{array}
$$

These may be written in form

$$
\int_{\mathfrak{X}^{(12 \cdots k-1)}} \Pi^{(12 \cdots k-1)} f_{j}^{(12 \cdots k-1)}=0 \quad(j=1,2, \cdots, m)
$$

the function $\Pi^{(12 \cdots k-1)}$ being defined on the range $\mathfrak{X}^{(12 \cdots k-1)}$ as follows:

$$
\Pi^{(12 \cdots k-1)} \equiv\left\{\begin{array}{c}
\int_{\mathfrak{X}_{P}^{(12 \cdots k)}} \Pi^{(12 \cdots k)}(p, n) f_{k}^{(12 \cdots k-1)}(p) d p \text { on } \mathfrak{X}_{N}(12 \cdots k), \\
-\int_{\mathfrak{X}_{N}^{(12 \cdots k)}} \Pi^{(12 \cdots k)}(p, n) f_{k}^{(12 \cdots k-1)}(n) d n \text { on } \mathfrak{X}_{P}^{(12 \cdots k)}
\end{array}\right.
$$


It is obvious from this definition that the function $\left.\Pi^{(12} \cdots k-1\right)$ is positive on the range $\mathfrak{X}^{(12 \cdots k-1)}$. It is likewise continuous at all points except possibly at those points of $\mathfrak{X}_{P}(12 \cdots k)$ which are limit points of $\mathfrak{X}_{N}(12 \cdots k)$.

In the next section we make an alteration in the procedure which will insure continuity at these points also.

6. An alteration to secure continuity. In the preceding section the function $\Pi^{(12 \cdots k)}$ is assumed to possess certain properties, and upon these assumptions the existence and certain properties of the function $\Pi^{(12 \cdots k-1)}$ are established. The function $\Pi^{(12 \cdots k)}$ of the hypothesis is still unnecessarily general for our purpose. By making certain additional assumptions with regard to it we may expect to obtain additional properties for the function $\left.\Pi^{(12} \cdots k-1\right)$. The additional property desired is continuity. To this end, we now assume that $\Pi^{(12 \cdots k)}$ is equal to unity at all points of the range $\mathfrak{X}^{(12 \cdots k)}$ except on some closed aggregates of inner points.

Now if $p_{0}$ is a point of $\mathfrak{X}_{P}{ }^{(12 \cdots k)}$ which is a limit point of $\mathfrak{X}_{N}(12 \cdots k)$, then $\left(p_{0}, n\right)$ is, for every $n$, a frontier point of $\mathfrak{X}^{(12 \cdots k)}$, and from the assumption just made it follows that $\Pi^{(12 \cdots k)}\left(p_{0}, n\right)=1$. Hence from the definition of $\Pi^{(12 \cdots k-1)}$,

$$
\Pi^{(12 \cdots k-1)}\left(p_{0}\right)=-\int_{\mathfrak{X}_{N}^{(12 \cdots k)}} f_{k}^{(12 \cdots k-1)}(n) d n .
$$

Furthermore, from the assumption it follows that for all points $n$ of $\left.\mathfrak{X}_{N^{(12}} \cdots k\right)$ sufficiently near to $p_{0}, \Pi^{(12 \cdots k)}(p, n)=1$, and hence for such values of $n$

$$
\Pi^{(12 \cdots k-1)}(n)=\int_{\mathfrak{X}_{P}^{(12 \ldots k)}} f_{k}^{(12 \cdots k-1)}(p) d p .
$$

Hence we secure continuity of $\Pi^{(12 \cdots k-1)}$ at all such points $p_{0}$ on the range $\mathfrak{X}^{(12 \cdots k-1)}$ if

$$
\int_{\left.\mathfrak{X}_{P}^{(12} \cdots k\right)} f_{k}^{(12 \cdots k-1)}(p) d p=-\int_{\left.\mathfrak{X}_{N}^{(12} \cdots k\right)} f_{k}^{(12 \cdots k-1)}(n) d n=1 .
$$

We secure this property (10) by prefixing a slight preparatory operation to the reduction process previously described.

In the set

$$
\left\{f_{3}^{(12 \cdots k-1)}\right\} \quad 0, \cdots, 0, f_{k}^{(12 \cdots k-1)}, \cdots, f_{m}^{(12 \cdots k-1)},
$$

the function $f_{k}^{(12 \cdots k-1)}$, specified as the next reducing function, may or may not satisfy the equality 


$$
\int_{\mathfrak{X}^{(12 \cdots k-1)}} f_{k}^{(12 \cdots k-1)}=0
$$

If this condition is satisfied, then

$$
\int_{\mathfrak{X}_{P}^{(12 \cdots k)}} f_{k}^{(12 \cdots k-1)}=-\int_{\mathfrak{X}_{N}^{(12 \cdots k-1)}} f_{k}^{(12 \cdots k-1)}=\text { a positive constant }
$$

and division of $f_{k}^{(12 \cdots k-1)}$ by this constant gives a new function by which it can be replaced, and the new function will have the property indicated by (10).

If the condition (10) is not satisfied by a constant times $f_{k}^{(12 \cdots k-1)}$ but the analogous condition is satisfied by a constant times some succeeding function in the sequence $\left\{f_{i}^{(12 \cdots k-1)}\right\}$, the two functions may be interchanged and the desired property secured.

Suppose then that no one of the functions $f_{i}^{(12 \cdots k-1)}(j \geqq k)$ satisfies the condition indicated by (10). Then we replace $f_{k}^{(12 \cdots k-1)}$ by a linear combination

$$
\bar{f}_{k}^{(12 \cdots k-1)}=\alpha f_{k}^{(12 \cdots k-1)}+\beta f_{k+1}^{(12 \cdots k-1)}
$$

where the constants $\alpha$ and $\beta$ are so chosen that the condition analogous to (10) is satisfied by the function $\bar{f}_{k}^{(12 \cdots k-1)}$. The replacement of $f_{k}^{(12 \cdots k-1)}$ by this function will not affect any of the vital properties of our reduction process, and its use secures the desired continuity of the function $\left.\Pi^{(12} \cdots k-1\right)$.

Furthermore, a consideration of the definition of $\Pi^{(12 \cdots k-1)}$ in the preceding section, together with the additional hypothesis on $\left.\Pi^{(12} \cdots k\right)$ in the present section and the condition (10), shows that the function $\Pi^{(12 \cdots k-1)}$ is equal to unity at all points of its range $\mathfrak{X}^{(12 \cdots k-1)}$ except some closed sub-sets composed entirely of inner points of the range. Hence we have the following amplification of the property (b) as stated in $\$ 1$ :

If all the functions of any reduced set admit a common orthogonal function which is positive, continuous, and equal to unity except on some closed sub-sets of inner points, then the set of functions from which it is obtained by reduction has the same property.

7. Conclusion of the proof of Theorem I. Starting with the given set of functions

$$
f_{1}(x), f_{2}(x), \cdots, f_{m}(x)
$$


we obtain, after $m-1$ reductions of the type described in the preceding sections, a set

$$
0, \cdots, 0, f_{m}^{(12 \cdots m-1)},
$$

in which all functions except the last one, $\left.f_{m}^{(12} \cdots m-1\right)$, are identically zero. If this last set admits a positive orthogonal function, continuous, and equal to unity except on closed sub-sets of inner points, then by $\$ 6$ the given set admits a positive and continuous orthogonal function, and the proof of our theorem is complete.

Our problem is then reduced to showing that the set $\left\{f_{i}^{(12 \cdots m-1)}\right\}$ admits an orthogonal function of the kind described, or more simply still, that the single function $\left.f_{m}^{(12} \cdots m-1\right)$ admits such an orthogonal function. This is not difficult.

From the hypothesis that every linear combination of the given functions changes sign, it follows by $\$ 4$ that $\left.f_{m}^{(12} \cdots m-1\right)$ changes sign internally. Therefore from the continuity preserved in the reduction process it follows that there is a set of inner points completely bounded by inner points on which the function is positive, and a similar set of points on which it is negative. We denote, for the moment, the former set by $\mathfrak{P}$ and the latter set by $\Re$, and consider two functions $\Pi_{p}$ and $\Pi_{n}$ on the range $\mathfrak{X}^{(12 \cdots m-1)}$, restricted by the following conditions. Both functions are continuous. The former $\Pi_{p}$ is positive on the region $\Re$ (excluding the boundary) and is elsewhere zero. The latter $\Pi_{n}$ is positive on the region $\mathfrak{N}$ (excluding the boundary) and is elsewhere zero.

Next we consider the function

$$
\Pi^{(12 \cdots m-1)} \equiv 1+\alpha \Pi_{p}+\beta \Pi_{n},
$$

$\alpha$ and $\beta$ being constants to be determined. This function is evidently continuous on $\mathfrak{X}^{(12 \cdots m-1)}$, and is positive if $\alpha$ and $\beta$ are positive. Furthermore it is equal to unity except at points of $\mathfrak{P}$ and $\Re$.

We now choose $\alpha$ and $\beta$ so that

$$
\int_{\left.\mathfrak{X}^{(12} \cdots m-1\right)} \Pi^{(12 \cdots m-1)} f_{m}^{(12 \cdots m-1)}=0,
$$

which is equivalent to

$$
\begin{aligned}
\int_{\left.\mathfrak{X}^{(12} \cdots m-1\right)} f_{m}^{(12 \cdots m-1)} & +\alpha \int_{\mathfrak{X}^{(12 \cdots m-1)}} \Pi_{p} f_{m}^{(12 \cdots m-1)} \\
& +\beta \int_{\left.\mathfrak{X}^{(12} \cdots m-1\right)} \Pi_{n} f_{m}^{(12 \cdots m-1)}=0 .
\end{aligned}
$$


Since the second integral is positive and the third integral is negative, $\alpha$ and $\beta$ can be given positive values which will satisfy this condition. The function $\left.\Pi^{(12} \cdots m-1\right)$ defined by (11) is then positive, continuous on $\mathfrak{X}^{(12 \cdots m-1)}$, and is equal to unity except at points of $\mathfrak{P}$ and $\Re$. It is orthogonal to $f_{m}^{(12 \cdots m-1)}$ and hence to all functions of the set $\left\{f_{i}^{(12 \cdots m-1)}\right\}$. From the existence of such a function there follows, as we have seen, the existence of a positive continuous function orthogonal to all functions of the given set $\left\{f_{i}\right\}$ and the proof of Theorem I is complete.

8. Linear integral inequalities. As an application of the theorem proved in the foregoing sections we consider the following problem.

Given the linear integral inequality

$$
\phi(x)+\int_{a}^{b} \kappa(x, s) \phi(s) d s>0
$$

in which the kernel $\kappa(x, s)$ is continuous on the square

$$
a \leqq x \leqq b, \quad a \leqq s \leqq b .
$$

Under what conditions will the inequality admit a solution $\phi(x)$ continuous on the interval $\mathfrak{X}$ ?

We note first that (12) is equivalent to an integral equation

$$
\phi(x)+\int_{a}^{b} \kappa(x, s) \phi(s) d s=\pi(x),
$$

where $\pi(x)$ is positive and continuous, but otherwise subject to determination.

If the Fredholm determinant $D$ of the kernel $\kappa(x, s)$ is different from zero, (13) possesses for any continuous $\pi(x)$ a continuous solution

$$
\phi(x)=\pi(x)-\frac{1}{D} \int_{a}^{b} D(x, s) \pi(s) d s
$$

where $D(x, s)$ is the first minor of $D$. Hence we have the result

If the Fredholm determinant $D$ of the kernel $\kappa(x, s)$ is different from zero, the general solution of the inequality (12) is given by the formula (14) in which $\pi(x)$ is positive and continuous but otherwise arbitrary.

In case $D=0$, the equation (13) for a given $\pi(x)$ in general admits no solution. A necessary and sufficient condition for the existence of a solution 
is that $\pi(x)$ be orthogonal to every solution of the associated homogeneous equation

$$
\psi(x)+\int_{a}^{b} \psi(s) \kappa(s, x) d s=0,
$$

or what is equivalent, that $\pi(x)$ be orthogonal to every one of a fundamental set of solutions of (15). Suppose such a set is

$$
\psi_{1}(x), \psi_{2}(x), \cdots, \psi_{m}(x) .
$$

Then the inequality (12) will have a solution if and only if the set of functions $\left\{\psi_{i}\right\}$ admits a positive function $\pi(x)$ orthogonal to all of them.

By our Theorem I, a necessary and sufficient condition for the existence of such a function $\pi(x)$ is that every linear combination of the functions of the set shall change sign. But the linear combinations of these functions constitute the non-trivial* solutions of the equation (15). Hence we have

Theorem II. A necessary and sufficient condition that the integral inequality (12) admit a solution $\phi(x)$ is that every non-trivial solution $\psi(x)$ of the associated integral equation (15) shall change sign. $\dagger$

* By a non-trivial solution we mean a solution which is not identically zero.

$\uparrow$ The case in which $D \neq 0$ is compatible with the theorem, since in that case the equation (15) admits no non-trivial solution, and the inequality (12) admits a solution (14).

UNIVERSTTY OF SASKATCHEWAN,

Sastatoon, Canada 